\title{
Associations of Job Demands and Patient Safety Event Involvement on Burnout among a Multidisciplinary Group of Pediatric Hematology/Oncology Clinicians
}

\author{
Tyler Dunn ${ }^{1}$, Michael Terao ${ }^{2}$, Lindsay Blazin ${ }^{3}$, Holly Spraker-Perlman ${ }^{4}$, Justin Baker ${ }^{4}$, \\ Belinda Mandrell ${ }^{4}$, Janet Sellers ${ }^{5}$, Valerie Crabtree ${ }^{6}$, James Hoffman ${ }^{4}$, and Jonathan \\ Burlison $^{5}$ \\ ${ }^{1}$ University of Mississippi \\ ${ }^{2}$ MedStar Georgetown University Hospital \\ ${ }^{3}$ Indiana University School of Medicine \\ ${ }^{4}$ St. Jude Children's Research Hospital \\ ${ }^{5}$ St Jude Children's Research Hospital \\ ${ }^{6}$ St Jude
}

April 7, 2021

\begin{abstract}
BACKGROUND: Workplace burnout can result in negative consequences for clinicians and patients. We assessed burnout prevalence and sources among pediatric hematology/oncology inpatient nurses, ambulatory nurses, physicians (MDs), and advanced practice providers (APPs) by evaluating effects of job demands and involvement in patient safety events (PSEs). METHODS: A cross-sectional survey (Maslach Burnout Inventory) measured emotional exhaustion, depersonalization, and reduced personal accomplishment. The NASA Task Load Index measured mental demand, physical demand, temporal demand, effort, and frustration. Relative weights analyses estimated the unique contributions of tasks and PSEs on burnout. Post-hoc analyses evaluated open-response comments for burnout factors. RESULTS: Burnout prevalence was 33\%, 20\%, 34\% and $33 \%$ in inpatient nurses, ambulatory nurses, and MD, and APPs respectively ( $\mathrm{N}=481$, response rate $69 \%$ ). Reduced personal accomplishment was significantly higher in inpatient nurses than MDs \& APPs. Job frustration was the most significant predictor of burnout across all four cohorts. Other significant predictors of burnout included temporal demand (nursing groups \& MDs) effort (inpatient nurses \& MDs) and PSE involvement (ambulatory nurses). Open-response comments identified time constraints, lack of administrator support, insufficient institutional support for self-care, and inadequate staffing and/or turnover as sources of frustration. CONCLUSIONS: All four clinician groups reported substantial levels of burnout, and job demands predicted burnout. The body of knowledge on job stress and workplace burnout supports targeting organizational-level sources, versus individual-level factors, as the most effective prevention and reduction strategy. This study elaborates on this evidence by identifying structural drivers of burnout within a multidisciplinary context of pediatric hematology/oncology clinicians.
\end{abstract}

\section{INTRODUCTION}

Burnout is a psychological syndrome that develops as a prolonged response to job-related stressors consisting of 3 key dimensions: emotional exhaustion, depersonalization, and reduced personal accomplishment. ${ }^{1}$ Causes and consequences of burnout are being increasingly recognized as an important social issue, especially in healthcare settings. ${ }^{2}$ A recent National Academy of Medicine publication reported that burnout among healthcare professionals negatively impacts healthcare quality and safety, decreases patient satisfaction, increases staff turnover, reduces work effort, and has negative financial implications for healthcare 
organizations. ${ }^{3}$

Roth et al. reported burnout in $38 \%$ of pediatric oncologists in the US, Canada, and 11 other countries. ${ }^{4}$ A meta-analysis of burnout rates among oncology nurses showed $30 \%, 15 \%$, and $35 \%$ having high emotional exhaustion levels, high depersonalization levels, and reduced personal accomplishment levels, respectively. ${ }^{5}$ Thus, it is crucial to identify contributing (or causative) workplace factors to develop effective interventions to prevent or reduce burnout among clinicians in oncology settings. ${ }^{6}$

Halbesleben and Rathert observed that in physicians, higher depersonalization levels were associated with lower patient satisfaction and increased hospitalization recovery time ${ }^{7}$ and Shanafelt and colleagues found that physicians with burnout were more likely to spend less time caring for patients. ${ }^{8}$ Surgeons with higher burnout levels had higher rates of self-reported medical errors $;^{9}$ emergency room physicians with higher burnout scores reported higher rates of suboptimal patient care (e.g., not treating a patient's pain in a timely manner $){ }^{10}$

Nurse burnout also negatively impacts patient care. Higher patient urinary tract infection and surgical site infection rates have been associated with burnout in nurses. ${ }^{11}$ Emotional exhaustion and reduced personal accomplishment among nurses was negatively associated with patient satisfaction, ${ }^{12}$ and higher burnout levels among nurses were associated with lower peer-rated quality of care. ${ }^{13}$

The impact of job demands and patient safety event (PSE) involvement on burnout have been previously studied among healthcare workers but not specifically in a multidisciplinary cohort of pediatric hematology/oncology clinicians. ${ }^{14}$ The study objectives were to: (1) assess the prevalence of burnout among inpatient and ambulatory care nurses, physicians (MDs) and advanced practice providers (APPs); (2) compare the differences in job demands, PSE involvement, and burnout rates across groups; and (3) determine the impact of job demands and PSE involvement on levels of burnout.

\section{METHODS}

\section{Study Setting, Design, and Participants}

St. Jude Children's Research Hospital is a 78-bed pediatric hospital with integrated outpatient clinics offering subspecialty and surgical services for children with cancer, blood disorders, and other catastrophic diseases. Approximately 7,500 patients are seen per year. Responses from a cross-sectional survey as part of a routine patient safety culture assessment were collected from MDs, APPs, and nurses in December 2018. Participants were recruited through email invitations from hospital leadership, newsletter announcements, digital signage, and mailed invitation postcards. Participants were categorized as inpatient nurses, ambulatory nurses, MDs and APPs. A subset of this study's data was used in a previous publication. ${ }^{15}$

\section{Study Measures}

The Maslach Burnout Inventory (MBI) Human Services Survey for Medical Personnel is a 22-item selfreported survey instrument developed to assess the 3 dimensions of burnout using a multidimensional model. ${ }^{1}$ Whereas previous measures of burnout focused only on the exhaustion dimension, ${ }^{16}$ the MBI incorporates the central construct of exhaustion and extends measurement to depersonalization and reduced personal accomplishment constructs to articulate interrelationships. ${ }^{17}$ The MBI is widely used in burnout research and considered the standard tool for measurement. ${ }^{18} \mathrm{MBI}$ items are scored by a 7-point frequency scale from "never" to "daily."

The MBI was not developed as a clinical diagnostic tool, and therefore does not distinguish between burnedout and not burned-out individuals. However, studies have attempted to validate MBI cutoff scores that reflect both a binary distinction of burnout, and also low, moderate, and high levels of emotional exhaustion, depersonalization, and reduced personal accomplishment. The MBI Manual (4th edition; 2016) removed cutoff scores due to lack of diagnostic validity. ${ }^{17}$ This study reported burnout levels only to compare with previous studies administering the MBI to participants within oncology settings. ${ }^{4,5}$ Therefore, high level burnout was 
defined when respondents had 1 of 3 criteria: raw sum score of [?]27 in the emotional exhaustion dimension, [?]10 in the depersonalization dimension, or [?]15 in the reduced personal accomplishment dimension. ${ }^{11,12,19,20}$

Workplace factors were measured with the National Aeronautics and Space Administration Task Load Index (NASA-TLX) ${ }^{21}$ which measures 5 aspects of subjective job demand: mental demand, physical demand, temporal demand, effort, and frustration. NASA-TLX scores ranged from 0 to 10; higher scores reflected higher perceived workload. The NASA-TLX has been previously used in healthcare research. ${ }^{14,22}$

A screener question from the Second Victim Experience and Support Tool (SVEST) was used to assess PSE involvement. The SVEST is a 29-item survey instrument designed to provide healthcare organizations information on PSE involvement support resources most preferred by clinicians. ${ }^{23}$ SVEST responses are recorded with 5-point Likert-scale agreement items ranging from "strongly disagree" to "strongly agree".

Participants could also include open-response comments via the item, "Please use this space to include any comments about how we can improve patient safety or the work experience for the St. Jude patient care staff." These comments were analyzed post-hoc, in response to results demonstrating associations among the NASA-TLX job demand, frustration, and burnout prevalence. Two authors (TD \& JDB) reviewed comments independently to identify broad concepts related to potential sources of frustration. Final concept terminology was developed by consensus, and content analysis methods identified relevant comments. Content analysis disagreements were resolved through consensus.

\section{Statistical Analyses}

MBI results were analyzed by descriptive statistics (Table 1). ANOVAs and Tukey's post hoc tests determined significant differences in job demand, involvement in PSEs, and burnout in inpatient nurses, ambulatory nurses, MDs and APPs. Statistical analyses were performed using SAS, version 9.4 (SAS Institute).

To evaluate associations among MBI dimensions and NASA-TLX job demands, relative weights analysis (RWA) was conducted using RWA-Web. ${ }^{24,25}$ Relative weight refers to the proportionate contribution of each predictor variable to the variance accounted for in a regression model while considering its unique contribution and that when combined with other variables. ${ }^{24}$ RWA is superior to traditional regression analysis as it generates a more accurate ranking of each predictor's unique contribution to an outcome variable. RWA provides accurate estimates of the relative importance of predictors in cases of high levels of collinearity. ${ }^{26}$ Our study conducted 9 RWA (3 cohorts $\times 3$ burnout dimensions). In all analyses, $95 \%$ confidence intervals for each individual relative weight and all corresponding significance tests were based on bootstrapping with 10,000 replications.

\section{RESULTS}

We received 481 complete survey responses (response rate $69 \%$ ). The final sample included $65 \mathrm{MDs}, 66 \mathrm{APPs}$, 239 inpatient nurses, and 111 ambulatory nurses. Subgroup response rates were $50 \% \%$ for MDs, $60 \%$ for APPs, $81 \%$ for inpatient nurses, and $68 \%$ for ambulatory nurses. Table 1 contains MBI descriptive statistics and percentages of respondents who qualified as experiencing high burnout.

\section{Differences in Job Demands, Patient Safety Event Involvement, and Burnout}

Table 1 summarizes results from each of the 3 MBI dimensions across the subgroups. Inpatient nurses reported significantly higher levels of reduced personal accomplishment than MDs and APPs $(p<.001)$. There were no other significant differences among subgroups in emotional exhaustion or depersonalization. Table 2 summarizes respondents' perceived job demands, and involvement in PSEs by subgroups. Mental demand was the highest ranked job demand among all 4 cohorts. We observed significant differences among cohorts in physical job demands: MDs \& APPs reported significantly lower physical demand than did inpatient nurses $(P<.001)$ and ambulatory nurses $(P<.001)$. Further, inpatient nurses reported significantly higher physical demands than did ambulatory nurses (6.1 vs. $5.3, P=.006)$. No significant differences were observed among the cohorts for PSE involvement.

\section{Relative Weight Analyses}




\section{Physicians}

Table 3 gives results of the RWAs. Among MDs, a weighted linear combination of 6 predictor variables included in the model explained more than $50 \%$ of the variance in emotional exhaustion $\left(R^{2}=0.53\right)$ but explained a lower proportion of variance in depersonalization $\left(R^{2}=0.30\right)$ and reduced personal accomplishment $\left(R^{2}=0.21\right)$. Frustration explained a statistically significant proportion of variance in the three burnout dimensions: $49.5 \%$ in emotional exhaustion, $87.9 \%$ in depersonalization, and $48.7 \%$ in reduced personal accomplishment. Effort (14.5\%) and temporal demand (20.0\%) also explained a significant portion of variance in MD emotional exhaustion.

Advanced Practice Providers

Among APPs, a weighted linear combination of 6 predictor variables included in the model explained more than half of the variance in emotional exhaustion $\left(R^{2}=0.62\right)$ but explained a lower proportion of variance in depersonalization $\left(R^{2}=0.35\right)$ and reduced personal accomplishment $\left(R^{2}=0.17\right)$. Frustration explained a statistically significant proportion of variance in the three burnout dimensions: $67.2 \%$ in emotional exhaustion, $72.4 \%$ in depersonalization, and $73.6 \%$ in reduced personal accomplishment.

Inpatient Nurses

Similar to providers, a weighted linear combination of the 6 predictor variables explained a high proportion of variance in emotional exhaustion among inpatient nurses $\left(R^{2}=0.55\right)$ but explained a smaller level of variance in depersonalization $\left(R^{2}=0.28\right)$ and reduced personal accomplishment $\left(R^{2}=0.19\right)$. Three variables explained a significant proportion of variance in emotional exhaustion with the most important variable being frustration ( $71.0 \%$ of variance), followed by temporal job demand ( $10.4 \%$ of variance), and effort job demand (7.9\% of variance). For depersonalization, frustration explained $60.2 \%$ of variance, PSE involvement explained $16.8 \%$, and temporal job demand explained $9.6 \%$. Only frustration significantly explained variance in reduced personal accomplishment among inpatient nurses with $79.8 \%$ of variance explained.

\section{Ambulatory Care Nurses}

Among ambulatory nurses, the 6 variables included in the model explained a higher proportion of variance in emotional exhaustion $\left(R^{2}=0.64\right)$ than depersonalization $\left(R^{2}=0.30\right)$ and reduced personal accomplishment $\left(R^{2}=0.12\right)$. Variables that explained a statistically significant amount of variance in emotional exhaustion included frustration (54.7\%), temporal job demand (16.2\%), and patient safety event involvement (12.4\%). Variance in depersonalization was significantly explained by two variables: frustration explaining $60.5 \%$ and mental job demand explaining $14.8 \%$ of the variance. As with the other cohorts' results, variance in reduced personal accomplishment was only significantly explained by frustration ( $87.5 \%$ of variance explained).

\section{Open Response Comments}

Post-hoc review of open-response comments identified 4 dominant categories of frustration sources: (1) Time constraints, (2) Lack of administrator support, (3) Insufficient psychosocial support resources, and (4) Inadequate staffing and/or turnover. Time constraint comments reflected perceptions of insufficient time to complete assigned tasks or feeling generally overwhelmed with expected workload. Lack of administrator support generally characterized desires for management to involve staff in policy development, process changes, and to provide greater support in conflict resolution with families, patients, or colleagues. Insufficient institutional support for self-care reflected the need for additional resources to process the emotional toll of providing care for seriously ill and dying children. Staffing and turnover frustrations were often described as threats to department-level culture or morale. See Table 4 for exemplar comments representing these themes.

\section{DISCUSSION}

Burnout prevalence and workplace factors that influence burnout were measured among clinicians who provide pediatric care in a hematology-oncology setting. Burnout levels were substantial across all 4 rolebased cohorts in all 3 MBI dimensions, similar to previous studies. ${ }^{10,11}$ To contextualize part of our results, respondents from all 4 cohorts reported feeling emotionally exhausted or experiencing reduced personal 
accomplishment from their work an average of twice per month. Depersonalization (e.g., treating a patient as an immaterial object) rates were substantially lower compared to emotional exhaustion and reduced personal accomplishment, which is encouraging as this construct represents a lack of presenteeism and may reflect a more severe expression of burnout. ${ }^{26}$

Among workplace factors associated with burnout, the NASA-TLX dimension, "frustration", was the most significant contributor across all 4 cohorts and 3 MBI dimensions. Temporal demand was also associated with burnout among MDs and nurses, but to much less a degree than frustration. Similarly, PSE involvement was significantly associated with burnout only among nurses.

Our post-hoc review of open-response comments elucidates the strong associations we observed between burnout rates and frustration levels. Comments addressing time constraints parallel our temporal demand results, and perceptions of feeling rushed or having inadequate time to complete tasks effectively are known predictors of burnout. ${ }^{28-30}$ Respondents expressed perceptions of insufficient administrator support in terms of feeling unable to garner managerial assistance when experiencing confrontations with colleagues, families, or patients. They also noted a general disconnect between the leadership's perspective and the realistic needs of front-line staff. Insufficient institutional support for self-care reflected a desire for time away from work after experiencing patient loss, including both an immediate reprieve from clinical duties and paid time off to grieve and recover. Finally, perceptions of inadequate staffing and frequent turnover were identified. Inadequate staffing can result in increased individual task demands (a commonly cited predictor of burnout), ${ }^{31}$ and restrict employees' ability to utilize paid time off. Turnover, especially voluntary turnover, can affect unit morale. ${ }^{32,33}$ When team members quit, remaining members may face a paucity of expertise and need to mentor new colleagues. Hence, inadequate staffing and turnover may create and perpetuate burnout in those perceiving a lack of adequate resources as an increase in individual demands is required to accomplish the necessary tasks.

PSE involvement can be detrimental to a clinician's psychological well-being decades after the event, and there are unfortunate examples of clinician suicide after medical errors. ${ }^{34}$ Effects can mirror post-traumatic stress disorder and include lingering negative perceptions of professional self-efficacy. ${ }^{35}$ Our study is one of the first to identify a connection between involvement in PSEs and burnout among inpatient and ambulatory nurses in a pediatric hematology-oncology setting. Given the known consequences of burnout that include job performance deficits and potential turnover, our study provides further evidence for the development and implementation of resources to preserve the psychological well-being of staff involved in medical errors and serious safety events.

One strength of our study was using RWA, which addresses concerns raised when researchers use unique variance accounted for in regression models to rank predictors by significance. ${ }^{36}$ The principal challenge is that predictor variables are often intercorrelated, which leads to flawed interpretations of traditional metrics to partition unique predictor variance. We treated the $3 \mathrm{MBI}$ dimensions as separate outcomes and used RWA to determine unique contributions of 6 predictors (NASA-TLX dimensions and PSE involvement).

As for limitations, St. Jude has a unique care model treating only a specialized population of patients. Our results may not generalize well to other pediatric hospitals caring for children with varied acute and chronic issues or to smaller pediatric oncology settings. Cross-sectional designs have many limitations, including causality, directionality, and biases originating from the study sample (e.g., responder bias, recall bias, and selection bias). Lastly, the MBI is the most widely used burnout survey instrument but does not include clinically validated cutoff scores. Publishers of the MBI do not advocate using results to determine burnout rates or support cutoff scores for low, moderate, and high levels of MBI dimensions. Researchers have developed their own MBI cutoff scores, but they vary widely. ${ }^{37}$ Lack of standardized MBI cutoff scores limits the ability to compare burnout rates across studies, which hinders its capability in expanding knowledge on causes, effects, and protective resources to reduce and prevent burnout.

\section{CONCLUSION}

Clinicians and their patients can suffer from the effects of workplace burnout. Our study expands the un- 
derstanding of burnout prevalence and influential workplace factors among a large multidisciplinary cohort of hematology/oncology clinicians. We observed burnout rates similar to clinician cohorts in other medical specialties. Frustration, temporal demands, and involvement in PSEs were all significantly associated with higher burnout rates. Interventions that focus on time constraints, administrative support, psychosocial support resources, and staffing may prevent and reduce burnout.

\section{Conflicts of Interest}

The authors have no conflict of interest to declare.

\section{Acknowledgements}

Vani Shanker, $\mathrm{PhD}$ for copyediting the manuscript

\section{REFERENCES}

1. Maslach C, Schaufeli WB, Leiter MP. Job Burnout. Annu Rev Psychol . 2001;52(1):397-422.

2. Maslach C. A Multidimensional Theory of Burnout. In: Theories of Organizational Stress . 1998:68-85.

3. Dyrbye LN, Shanafelt TD, Sinsky CA, et al. Burnout Among Health Care Professionals: A Call to Explore and Address This Underrecognized Threat to Safe, High-Quality Care. NAM Perspectives . Published online July 5, 2017.

4. Roth M, Morrone K, Moody K, et al. Career burnout among pediatric oncologists. Pediatr Blood Cancer . 2011;57(7):1168-1173.

5. Fuente GAC-D la, Gómez-Urquiza JL, Ortega-Campos EM, Canadas GR, Albendin-Garcia L, FuenteSolana EID la. Prevalence of burnout syndrome in oncology nursing: A meta-analytic study. Psychooncology . 2018;27(5):1426-1433.

6. Jha AK, Iliff A, Chaoui AA, Defossez S, Bombaugh M, Miller Y. A crisis in health care: a call to action on physician burnout.Massachusetts Medical Society, Harvard TH Chan School of Public Health, and Harvard Global Health Institute. Published online 2019.

7. Halbesleben JRB, Rathert C. Linking physician burnout and patient outcomes: exploring the dyadic relationship between physicians and patients. Health Care Manage Rev . 2008;33(1):29-39.

8. Shanafelt TD, Mungo M, Schmitgen J, et al. Longitudinal Study Evaluating the Association Between Physician Burnout and Changes in Professional Work Effort. Mayo Clin Proc . 2016;91(4):422-431.

9. Shanafelt TD, Balch CM, Bechamps G, et al. Burnout and medical errors among American surgeons. Ann Surg . 2010;251(6):995-1000.

10. Lu DW, Dresden S, McCloskey C, Branzetti J, Gisondi MA. Impact of Burnout on Self-Reported Patient Care Among Emergency Physicians. West J Emerg Med . 2015;16(7):996-1001.

11. Cimiotti JP, Aiken LH, Sloane DM, Wu ES. Nurse staffing, burnout, and health care-associated infection. Am J Infect Control . 2012;40(6):486-490.

12. Vahey DC, Aiken LH, Sloane DM, Clarke SP, Vargas D. Nurse Burnout and Patient Satisfaction. Med Care . 2004;42(2 Suppl):II57-II66.

13. Poghosyan L, Clarke SP, Finlayson M, Aiken LH. Nurse burnout and quality of care: Cross-national investigation in six countries. Res Nurs Health . 2010;33(4):288-298.

14. Portoghese I, Galletta M, Coppola RC, Finco G, Campagna M. Burnout and Workload Among Health Care Workers: The Moderating Role of Job Control. Saf Health Work . 2014;5(3):152-157.

15. Blazin LJ, Terao MA, Spraker-Perlman H, Baker JN, Crabtree VM, Mandrell BN, Sellers J, Dunn TJ, Lu Z, Hoffman JM, Burlison JD. Never Enough Time: Mixed Methods Study Identifies Drivers of Temporal 
Demand That Contribute to Burnout Among Physicians Who Care for Pediatric Hematology-Oncology Patients. JCO Onc Pract. Published online: March 15, 2021.

16. Shirom A, Melamed S. A Comparison of the Construct Validity of Two Burnout Measures in Two Groups of Professionals. Int J Stress Manag . 2006;13(2):176-200.

17. Maslach C. Burnout: A Multidimensional Perspective. In:Professional Burnout: Recent Developments in Theory and Research . Theories of Organizational Stress. 1993:19-32.

18. Schaufeli WB, Enzmann D, Girault N. Measurement of burnout: A review. In: Professional Burnout: Recent Developments in Theory and Research . Series in applied psychology: Social issues and questions. Taylor \& Francis; 1993:199-215.

19. Maslach C, Jackson S, Leiter MP. Maslach Burnout Inventory Manual . 4th ed. Consulting Psychologists Press; 1996. Accessed March 10, 2020. https://www.mindgarden.com/maslach-burnout-inventory/685-mbimanual.html

20. Maslach C, Leiter MP. Early predictors of job burnout and engagement. J Appl Psychol . 2008;93(3):498512.

21. Hart SG, Staveland LE. Development of NASA-TLX (Task Load Index): Results of Empirical and Theoretical Research. In: Hancock PA, Meshkati N, eds. Advances in Psychology . Vol 52. Human Mental Workload. North-Holland; 1988:139-183.

22. McManus I, Keeling A, Paice E. Stress, burnout and doctors' attitudes to work are determined by personality and learning style: A twelve year longitudinal study of UK medical graduates. BMC Med . 2004;2:29.

23. Burlison JD, Quillivan RR, Scott SD, Johnson S, Hoffman JM. The Effects of the Second Victim Phenomenon on Work-Related Outcomes: Connecting Self-Reported Caregiver Distress to Turnover Intentions and Absenteeism. J Patient Saf . Published online November 2, 2016.

24. Johnson JW. A Heuristic Method for Estimating the Relative Weight of Predictor Variables in Multiple Regression. Multivariate Behav Res . 2000;35(1):1-19.

25. Tonidandel S, LeBreton JM. RWA Web: A Free, Comprehensive, Web-Based, and User-Friendly Tool for Relative Weight Analyses. J Bus Psychol . 2015;30(2):207-216.

26. Lebreton JM, Ployhart RE, Ladd RT. A Monte Carlo Comparison of Relative Importance Methodologies: Organ Res Methods . Published online June 29, 2016.

27. Maslach C, Leiter MP. Understanding the burnout experience: recent research and its implications for psychiatry. World Psychiatry . 2016;15(2):103-111. doi:10.1002/wps.20311

28. Bridgeman PJ, Bridgeman MB, Barone J. Burnout syndrome among healthcare professionals. Am J Health Syst Pharm . 2018;75(3):147-152.

29. Teng C-I, Shyu Y-IL, Chiou W-K, Fan H-C, Lam SM. Interactive effects of nurse-experienced time pressure and burnout on patient safety: A cross-sectional survey. Int J Nurs Stud . 2010;47(11):1442-1450.

30. Harolds JA, Parikh JR, Bluth EI, Dutton SC, Recht MP. Burnout of Radiologists: Frequency, Risk Factors, and Remedies: A Report of the ACR Commission on Human Resources. J Am Coll Radiol . 2016;13(4):411-416.

31. Bakker, AB, Demerouti, E, Verbeke, W. Using the Job Demands-Resources model to predict burnout and performance. J Hum Resour. 2004;43:83-104.

32. Hayes LJ, O'Brien-Pallas L, Duffield C, et al. Nurse turnover: a literature review. Int J Nurs. 2006;43(2):237-263. 
33. Misra-Hebert AD, Kay R, Stoller JK. A review of physician turnover:

rates, causes, and consequences. Am J Med Qual . 2004;19:56-66.

34. Seys D, Wu AW, Gerven EV, et al. Health Care Professionals as Second Victims after Adverse Events: A Systematic Review. Eval Health Prof . 2013;36(2):135-162..

35. Burlison JD, Scott SD, Browne EK, Thompson SG, Hoffman JM. The second victim experience and support tool (SVEST): Validation of an organizational resource for assessing second victim effects and the quality of support resources. J Patient Saf . 2017;13(2):93-102.

36. Tonidandel S, LeBreton JM. Relative Importance Analysis: A Useful Supplement to Regression Analysis. J Bus Psychol . 2011;26(1):1-9.

37. Doulougeri K, Georganta K, Montgomery A. "Diagnosing" burnout among healthcare professionals: Can we find consensus? Cogent Med . 2016;3(1):1

\section{Hosted file}

Dunn et al Table 1.pdf available at https://authorea.com/users/406432/articles/517092associations-of-job-demands-and-patient-safety-event-involvement-on-burnout-among-amultidisciplinary-group-of-pediatric-hematology-oncology-clinicians

\section{Hosted file}

Dunn et al Table 2.pdf available at https://authorea.com/users/406432/articles/517092associations-of-job-demands-and-patient-safety-event-involvement-on-burnout-among-amultidisciplinary-group-of-pediatric-hematology-oncology-clinicians

\section{Hosted file}

Dunn et al Table 3.pdf available at https://authorea.com/users/406432/articles/517092associations-of-job-demands-and-patient-safety-event-involvement-on-burnout-among-amultidisciplinary-group-of-pediatric-hematology-oncology-clinicians

\section{Hosted file}

Dunn et al Table 4.pdf available at https://authorea.com/users/406432/articles/517092associations-of-job-demands-and-patient-safety-event-involvement-on-burnout-among-amultidisciplinary-group-of-pediatric-hematology-oncology-clinicians 